\title{
Narrative review of palliative care in trauma and emergency general surgery
}

\author{
Alexandra C. Ferre ${ }^{1}$, Belinda S. DeMario ${ }^{2}$, Vanessa P. Ho Ho,3 $^{2,3}$ \\ ${ }^{1}$ Department of General Surgery, Digestive Disease Institute, Cleveland Clinic Foundation, Cleveland, Ohio, USA; ${ }^{2}$ Department of Surgery, \\ MetroHealth Medical Center, Cleveland, Ohio, USA; ${ }^{3}$ Department of Population and Quantitative Health Sciences, Case Western Reserve \\ University School of Medicine, Cleveland, Ohio, USA \\ Contributions: (I) Conception and design: All authors; (II) Administrative support: None; (III) Provision of study materials or patients: None; (IV) \\ Collection and assembly of data: All authors; (V) Data analysis and interpretation: All authors; (VI) Manuscript writing: All authors; (VII) Final \\ approval of manuscript: All authors. \\ Correspondence to: Vanessa P. Ho, MD, MPH, FACS. Division of Trauma, Critical Care, Burns, and Acute Care Surgery, Department of Surgery, 2500 \\ MetroHealth Drive, Cleveland, OH 44109, USA. Email: vho@metrohealth.org.
}

\begin{abstract}
Objective: The purpose of this article is to discuss the goals of palliative care with regards to acute care surgery patients and review the literature regarding administration and implementation of palliative programs.

Backgroundk For patients who experience unexpected and sometimes catastrophic life changes related to trauma or emergency general surgery, palliative care is a crucial adjunct that can help ensure the provision of optimal symptom management, communication, and goal-concordant care provided.

Methods: Palliative care is medical specialty with a philosophy of care focused on improving the quality of life for patients with serious injury or illness and their loved ones. Palliative care provides significant benefit across the entire spectrum of illness and injury, regardless of prognosis. We will discuss palliative care topics related to trauma and emergency general surgery patients, including symptom management, goal setting, end of life care, communication strategies, addressing implicit/explicit bias, trauma-specific and emergency general surgery-specific considerations, and implementation strategies to reduce barriers for utilization of palliative care.

Conclusions: Unfortunately, palliative care is often underutilized in the trauma and emergency general surgery population. Acute care surgeons should be familiar with principles of primary palliative care, as well as understand the added benefits that be provided by consultant palliative care specialists.
\end{abstract}

Keywords: Palliative care (PC); trauma surgery; emergency general surgery

Submitted Dec 04, 2020. Accepted for publication Aug 23, 2021.

doi: 10.21037/apm-20-2428

View this article at: https://dx.doi.org/10.21037/apm-20-2428

\section{Introduction}

The provision of appropriate, high-quality palliative care is an increasing focus of national healthcare initiatives (1). Palliative care (PC) is medical specialty with a philosophy of care focused on improving the quality of life for patients with serious injury or illness and their loved ones (2). While commonly thought to be primarily for those at or near the end of life, in reality palliative care provides significant benefit across the entire spectrum of illness and injury, regardless of prognosis (2). It is important to remember that any intervention aimed at easing patient pain and suffering falls under the category of palliative care. Many care settings may lack specialist palliative care to meet the needs of the emergency surgical population (3). Accordingly, trauma and surgical critical care providers must fill this gap by developing and utilizing primary palliative care skills.

Acute care surgery encompasses emergency general 
surgery (EGS), trauma, and critical care. Palliative care has been proposed as the "fourth pillar" of acute care surgery. In this model, ownership of surgical palliative care as a service provided by acute care and trauma surgeons ensures that critically injured patients receive optimal patient-centered care (4). Critical care providers should strive to integrate palliative medicine within daily intensive care services to facilitate goal-concordant treatment (4). However, many of these patients are complex and when available and applicable, consultant palliative care specialists are highly valuable members of a multidisciplinary team for seriously ill acute care surgery patients. In the same way that acute care surgeons provide critical care with a broadbased knowledge of renal and cardiac physiology but call consultants such as nephrology and cardiology for specific circumstances or disease processes, consultation of palliative care specialists can provide thoughtful and essential perspectives for patients with a high burden of symptoms or complex palliative needs.

In its broadest sense, palliative care can be viewed as the intensivist's tool to diagnose and treat suffering (5). Especially in a surgical critical care setting, providers can integrate palliative care through symptom management, most often including the treatment of pain, delirium, dyspnea, nausea and vomiting, fatigue, fluid overload, and anxiety, as well as through the elicitation of patient preferences and the organization of family meetings (6). Additionally, in especially serious cases, providers may also be responsible for discussing and implementing end of life care. Overall, the goal is to medically treat patients to ease their physical symptoms while also serving as an open line of communication between patients, family members and consulting teams.

In this narrative review, we: (I) discuss the goals, principles, and practice of palliative care with regards to trauma and emergency general surgery patients, including symptom management, goal setting and communication strategies, and addressing implicit/explicit bias; (II) review the literature regarding administration and implementation of palliative care programs for acute care surgery patients; (III) examine implementation strategies to reduce barriers for utilization of palliative care.

We present the following article in accordance with the Narrative Review reporting checklist (available at https://apm. amegroups.com/article/view/10.21037/apm-20-2428/rc).

\section{Background}

While there is increasing recognition of the importance of integrating palliative care with acute care surgery, it is not as simple as placing a consult for palliative care for every sick patient (7). Unfortunately, there is a systemic scarcity of specialist palliative care providers, with only one specialist palliative care provider per 1,200 seriously ill patients, and even fewer surgeons who are fellowship-trained in palliative care $(1,3)$. Due to this shortage, it is important for emergency and trauma surgeons and surgical intensivists bridge the gap and integrate palliative care interventions into their daily practice.

Unfortunately, there is currently a significant cultural gap between the acute care surgery community and the palliative care community. Acute care surgeons should aspire to be skilled at primary palliative care, or the use of basic palliative principles by a patient's primary provider, and provide symptom management and direct patients towards goal-concordant care for every patient, with a low threshold to consult specialist palliative medicine providers if additional needs are identified. Palliative care and acute care surgery can be mutually enhancing, rather than mutually exclusive to prioritize patient needs and align outcomes with patient goals.

Trauma and surgical ICUs present some of the most difficult challenges for integration of palliative care (6). In these high acuity units, aggressive resuscitation is an automatic response, and patients are often young and previously healthy. While the mortality rate for critically injured patients averages $10 \%$ to $20 \%$, these patients and their families are often unprepared for a catastrophic illness, and surgical ICU providers are prone to focus intensely on cure-oriented care $(6,8,9)$. Current literature reveals that high-quality palliative care in the ICU does not increase ICU or hospital mortality and instead shortens length of stay $(6,10,11)$. Some data suggest that early integration of palliative care in patients with serious illness may prolong meaningful life $(6,12)$. For many ICUs, cultural and logistical changes must be made in order to effectively implement palliative care interventions into the daily workflow.

\section{Methods}

The structure of this narrative review was agreed upon by all authors to cover the following topics: symptom management, family meetings and end-of-life care, communication strategies and frameworks, bias and inequity, trauma-specific considerations, emergency general surgery considerations, and implementation. The authors 
drafted the sections, supported by inductive PubMed searches for relevant publications and manual searches of bibliographies to identify key literature for each topic. All years of available years of PubMed were searched through November 2020, with a focus on English language articles.

\section{Narrative review of palliative care themes}

\section{Symptom management}

Symptom management plays an increasingly important role in the overall approach to seriously ill trauma and acute care surgical patients, especially in those who are acutely ill and in the ICU. Studies conducted in various ICU settings confirm that pain is the most prevalent and distressing physical symptom experienced by critically ill patients (13). Attention should be paid to pain control utilizing a variety of modalities. Treatments to relieve symptoms must take into account the complex pharmacologic and physiologic issues that accompany multiple organ failures (13).

Concerns about secondary effects of certain treatments, including hypotension, sedation, respiratory depression, delirium, and other alterations of consciousness, further complicate patient management (13). Opioids remain the mainstay of pain management in ICU patients, but nonopioid analgesics can be used in the ICU to supplement opioid pain control, including orally or intravenously administered acetaminophen; intravenously administered ketamine; and orally or intravenously administered antiinflammatory medications. For patients with neuropathic pain, gabapentin or carbamazepine can be considered (13). Regional nerve blockade for those with specific physical areas of pain can also be helpful, such as epidural catheters for patients with rib fractures. These pain interventions should be promptly made by the primary critical care team, and ongoing assessment of patient pain should be integrated into daily rounding protocols.

In addition to pain, other symptoms such as dyspnea can cause significant discomfort. Dyspnea is treated by optimizing the underlying etiological condition along with drug and non-drug treatments (13). Opioid medications remain the mainstay first-line drug of choice for treating dyspnea, but it is important to also correct underlying causes (13). Fluid overload is a common cause of dyspnea for patients who have received large volume resuscitations, and diuretics are commonly utilized to improve fluid balance and ease patient breathing. Supplemental oxygen and/or mechanical ventilation, invasive or noninvasive, can reduce dyspnea from respiratory failure, but may not be well tolerated in all patients (13). As stated by the Society of Critical Care Medicine's task force, the appropriate end point for use of supplemental oxygen and ventilation, invasive or non-invasive, with a critically ill patient whose treatment is focused exclusively on comfort is symptom relief; therefore, failure to improve or patient distress warrants discontinuation of mechanical ventilation (13). Positioning can be an important non-pharmacologic treatment for ICU patient's with dyspnea. For example, dyspnea in COPD can be reduced by upright positioning with arms elevated on pillows or a bedside table $(13,14)$. In unilateral lung disease, a sidelying position may be optimal to increase perfusion and/or ventilation (13).

Nausea and vomiting can also cause patients discomfort in the ICU. Controlling nausea and vomiting can help improve patient comfort (15). Antiemetics should be prescribed orally if there is nausea alone with vomiting. If there is significant vomiting, intravenous antiemetics can be prescribed. For persistent symptoms, an antiemetic should be prescribed regularly with a second line antiemetic prescribed on an "as required" basis. It is also important to identify the underlying cause of the nausea and/or vomiting and address this root cause. If the root cause of nausea and vomiting is increased intracranial pressure or a malignant bowel obstruction, dexamethasone can be used in addition to antiemetics. When a patient has significant vomiting, careful attention must be paid to hydration status and intravenous hydration titrated appropriately.

Mental status changes are also a major issue in the ICU setting. Addressing a patient's delirium and anxiety is an important part of critically care focused palliative care. Delirium is extremely common in patients in advanced phases of illness and represents one of the most frequent complication encountered in palliative care (16). Literature estimates that 30 to $50 \%$ of the delirium cases occurring in palliative care are reversible, making it an important intervention point (16). The primary goal of delirium treatment is the reversal of etiological factors $(16,17)$. In palliative care, opioid rotation, removal of any drug that is contributing to delirium, and management of clinical situations, such as dehydration and hypercalcemia, are the main principles of practice (17). Pharmacologic intervention occurs most often in the form of antipsychotic medications (16).

Similarly, anxiety commonly presents in critical care patients. The ICU setting is a high stress environment, and patients frequently feel anxious and unsettled (18). Additionally, the news of a short prognosis can increase 
patient anxiety and even trigger depression; therefore, close assessment and monitoring of patients' mood is imperative in the critical care setting. Interventions may consist of pharmacological treatments such as antidepressants or anxiolytics or non-pharmacological treatments such as talk therapy, music therapy or aromatherapy (18-20). Thoughtful family meetings and discussions can also help reduce anxiety by providing answers, knowledge, and empathy about the patient's medical condition.

\section{Family meetings and end-of-life care}

Both severe trauma and unplanned emergency surgery conditions are unexpected and sudden disease processes that can have devastating and life-threatening trajectories. Emotional adjustment to a devastating prognosis often fails to keep pace with illness progression, especially if the patient is young and was previously in good health, leaving patients and families confused and often contributing to extended futile care (6). High expectations for recovery can create a special challenge for establishing medically feasible goals of care (6).

Even under optimal conditions, the trauma or intensive care experience is burdensome for all patients and families, making care discussions more difficult (5). Over two-thirds of family members visiting a hospitalized relative in the ICU report anxiety and depression after the experience, and literature cites the most common palliative care recommendations were directed at improving provider communication with the family $(5,20,21)$. Due to the large impact of families on patient hospital experiences, familycentered care has emerged as a comprehensive ideal for navigating traumatic situations and managing end-oflife care (7). In fact, the presence and content of a family meeting documented in the medical record is in itself a quality measure of increasing focus (5).

A recent study by Bhangu and colleagues revealed that an early family meeting within 72 hours of trauma admission led to reduced ICU days, ventilator days, death during a code (22). In particular, multidisciplinary meetings were also associated with earlier transition to comfort care status (22). Since up to one-fourth of critically ill patients in the U.S. are likely to die within months of initial ICU admission, it makes intuitive sense to address the possibility of death early in the course of illness (5). An initial proactive family meeting with regular follow-up family meetings that revisit goals and expectations helps connect patients with loved ones and results in higher family satisfaction with care and increased comfort measures for patients (2). While clinical teams may focus on single items such as the donot-resuscitate (DNR) order or a therapeutic withdrawal, the family focus is a continuous process. While conducting family meetings, it is important to identify goals of care and expectations (23). Additionally, religious beliefs, ethnicity, and cultural background not only of the patients but also the providers may affect recommendations provided, and heightened physician awareness and a multidisciplinary approach is warranted (24). Rationale and strategies for recognizing and mitigating implicit bias in family meetings and end of life care are discussed below.

Literature has clearly shown advancing age is independently associated with withdrawal of life-sustaining measures in trauma patients (25). However in a retrospective review of elderly trauma patients, Trunkey et al. failed to show that age, maximum abbreviated injury score (AIS), comorbidities, admission Glasgow Coma Scale or vital signs were predictive of withdrawal of life sustaining therapy (26). This suggests that there may be different considerations for comfort care decisions amongst older trauma patients. Manara $e t a l$. revealed that pre-morbid activity, concern regarding loss of independence, and imminent death are all significant considerations in end of life decision making for older trauma patients, while quality of life considerations are the most significant factors for younger patients (27). For the critical care physician, understanding patients' goals of care and pre-injury functioning is important. It is important to determine unacceptable health states that no longer align with the patient' wishes or goals and use this as a transition point to comfort measures. The point where care transitions to a primary focus on comfort measures is different for each patient, and it often falls on the primary critical care physician to determine that point.

In addition to understanding patients' goals of care, providers must consider the skill set of the team providing care in end-of-life scenarios. Nursing staff play a key, and often underappreciated, role in the withdrawal of life support as they are at the bedside more than any other member of the critical care team and physically implement the orders to withdraw life support (28). One study showed that only $15.5 \%$ of 463 ICU nurse respondents had a required course that covered withdrawal of lifesustaining measures as part of their nursing curriculum, and $>63 \%(292 / 463)$ reported that they had no training for withdrawal of life-sustaining measures during orientation. Enhancing end-of-life training and support for ICU nurses is an important part of providing trauma patients with 
comprehensive care $(5,28)$.

By conducting culturally sensitive, multidisciplinary and compassion-based family meetings early following trauma admission, critical care physicians can improve patient care and ease familial burdens. Literature supports a comprehensive and honest family meeting within 72 hours of trauma admission and supports the integration of multiple care teams (2). All hospital systems should assess their family meeting protocols and incorporate changes that support prompt and inclusive meetings.

\section{Communication strategies and frameworks}

Communication with seriously ill or gravely injured patients in the acute setting is difficult. Communication frameworks, decision making aids and discussion protocols can help critical care providers utilize shared decision making to guide patient treatment plans. These methods prepare providers for difficult situations and allow for a more systematic approach to patient and family communication. There are many described frameworks, but we present three here, illustrated in Figure 1.

Cooper et al. explored the use of a communication framework to assist surgeons and critical care providers in delivering goal-concordant care for high-risk patients (23). This framework delineates 9 key elements: (I) formulating prognosis, (II) creating a personal connection, (III) disclosing information regarding the acute problem in the context of the underlying illness, (IV) establishing a shared understanding of the patient's condition, (V) allowing silence and dealing with emotion, (VI) describing surgical and palliative treatment options, (VII) eliciting patient's goals and priorities, (VIII) making a treatment recommendation, and (IX) affirming ongoing support for the patient and family. By structuring communication with critically ill patients and family, providers are able to systematically address key issues while maintaining open dialog. Using a communication framework provides structure to difficult discussions and helps to promote understanding and shared decision making.

Kruser et al. formulated a decision aid called "Best Case Worst Case" (29). They postulate that for patients who develop life-threatening surgical conditions, preoperative decision making is complex (29). In these situations, the "right" decision can only be reached by exploring each individual patient and family's goals and values. Essential tool elements include depiction of two or more treatment choices, creation of a pen-and-paper graphic aid, use of narrative to tell a story about how the patient might experience the outcomes in the best and worst case scenarios, estimation about the most likely outcome, description of how the treatment option impacts the larger context of the patient's overall health, and providing a treatment recommendation at the conclusion of the discussion (29). During the treatment conversation with the patient, the provider uses narrative to describe the best and worst possible outcomes of each treatment option, as well as the likely outcome, and creates a graphic aid to illustrate the range and estimated probability of each outcome (29).

This tool allows providers to illustrate the range of outcomes stemming from an injury or disease both narratively and pictorially to aid in patient and family comprehension. Additionally, the creation of a graphic aid allows the provider to give the family unit time to process and deliberate with a physical representation of the discussion. Another advantage of this method is that the provider can initiate the conversation before a definitive prognosis is known and when the outcomes uncertain. The "Best Case Worst Case" graphic can be updated as the patient's clinical situation develops.

Baile et al. describe a protocol for disclosing unfavorable information or "breaking bad news" to critically injured or ill patients called the SPIKES protocol (30). The protocol consists of six steps with the goal to enable the clinician to fulfill the four most important objectives of the interview disclosing bad news: gathering information from the patient, transmitting the medical information, providing support to the patient, and eliciting the patient's collaboration in developing a strategy or treatment plan for the future (30). The SPIKES protocol consists of six steps: (I) $\mathrm{S}$-setting up the interview, (II) $\mathrm{P}$-assessing the patient's perception, (III) I-obtaining the patient's involvement, (IV) $\mathrm{K}$-giving the knowledge and information to the patient, (V) E-addressing the patient's emotions with empathetic responses, (VI) S-strategy and summary. This protocol presupposes a dynamic interaction between physician and patient in which the clinician is guided by patient understanding, preferences, and behavior. It is a flexible approach, and as such, is more likely to address the inevitable differences among patients than a rigid recipe that is one size fits all (30).

\section{Addressing implicit or explicit bias/inequities}

A key aspect of palliative care in acute care surgery is quickly and peacefully establishing comfort measures in medically 

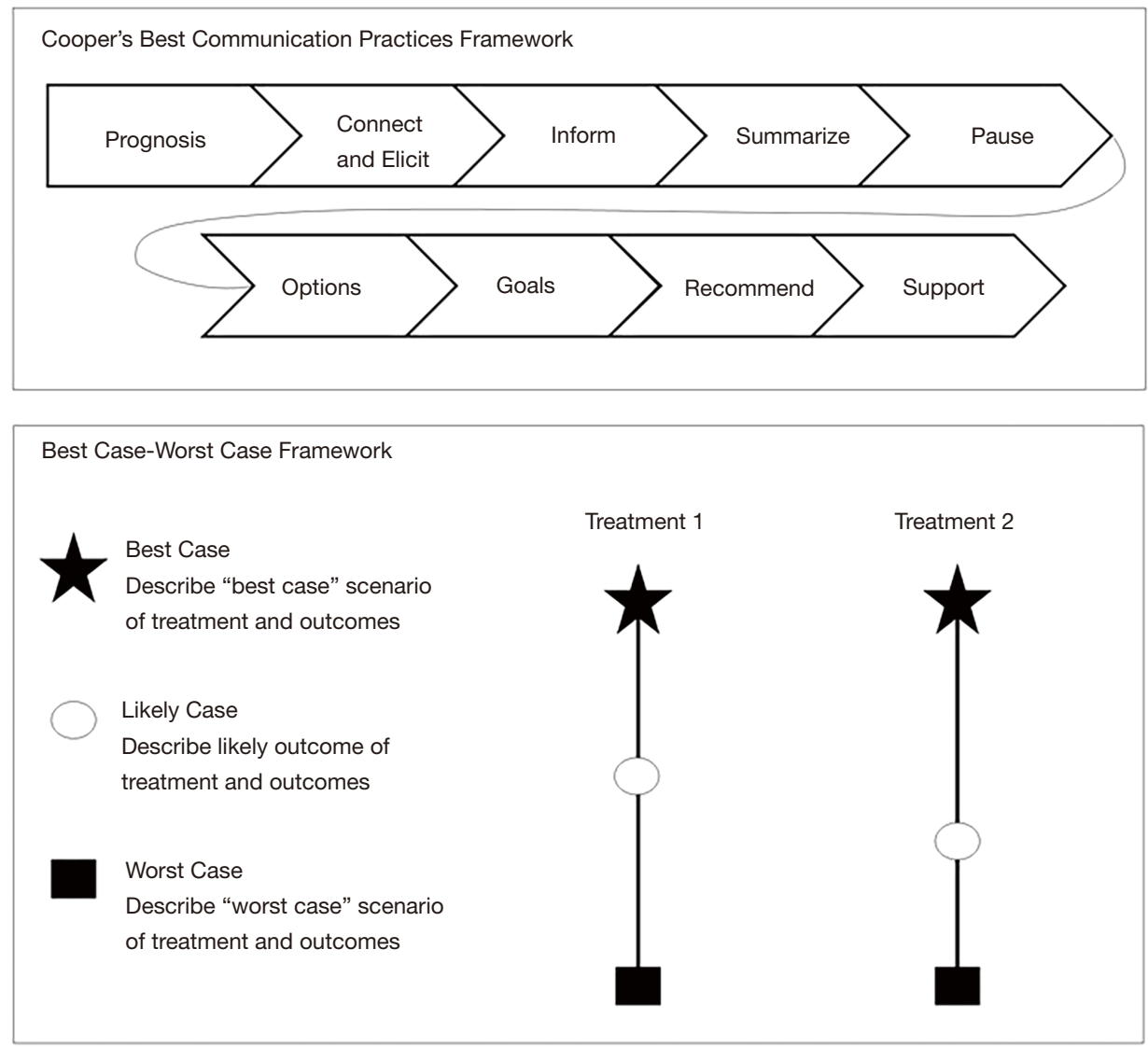

\begin{tabular}{|l|l|}
\hline SPIKES Six Step Protocol for Delivering Bad News \\
\begin{tabular}{|l|l|}
\hline S & Setting up the interview \\
\hline P & Assessing the patient's perception \\
\hline I & Obtaining the patient's involvement \\
\hline K & Giving knowledge and information to the patient \\
\hline E & Addressing the patient's emotions with empathetic responses \\
\hline S & Strategy and Summary \\
\hline
\end{tabular}
\end{tabular}

Figure 1 Three communication frameworks.

futile cases. While current medical systems aim to provide equitable care to all patients, inherent bias exists, and equal care is not readily available to all. Minding individual and system biases is an important part of the critical care physician's job, especially in palliative care situations. When time from admission to time to withdrawal of life-sustaining measures increases in critically injured patients, so does the potential for ineffective care, health care resource loss, and patient and family suffering (31).
A study by Hornor and colleagues found AfricanAmerican and Hispanic race are both significant predictors of late withdrawal of life-sustaining measures (31). Similarly, Haines et al. found race and ethnicity are independent predictors of a trauma patient's transition to hospice care. They demonstrated prominent racial and socioeconomic disparities exist, with uninsured and minority patients being less likely to receive hospice services and having a delay in transition to hospice care when compared to their 
insured Caucasian counterpart (32). Critical care physicians must pay special attention to patients from underserved populations. These patients are at risk for bias and poor delivery of care within the current healthcare system, and providers at the frontlines of treatment should take extra care to confront these biases to provide timely and appropriate palliative care. Understanding implicit bias is the first step towards righting a broken system and ensuring equitable care for all patients. Proactive policies to support equitable care would be the next steps, including use of standardized and regular reporting of race and ethnicity quality metrics, provision of financial support for equity initiatives, and community engagement of stakeholders for palliative care utilization.

\section{Trauma-specific considerations}

Guidelines on approaching palliative care discussions for trauma patients are available. The Trauma Quality Improvement Program (TQIP) has released Palliative Care Best Practices Guidelines with the goal of helping the trauma community implement this philosophy and care (2). These guidelines focus on integrating palliative measures such as symptom assessment and treatment, communication, family meetings and healthcare agent appointment into the ICU workflow for a trauma or surgical ICU. They focus on the development of a palliative care multidisciplinary team and cite communication as the backbone of the guidelines.

Communication should begin immediately with the establishment of family support. This should consist of an introduction to the multidisciplinary team, team sensitivity training, bereavement support preparation, and grief support availability. A comprehensive palliative care screen has been developed by The Palliative Care Network and adapted for trauma (33). It includes guidelines on early assessment, 72-hour assessment, and a comprehensive assessment. Providers should keep in mind that the unit of care is defined by TQIP is the "patient and the family" (2). Keeping this in mind can help guide interventions and meetings.

An early assessment should happen within 24 hours, addressing the following items with the patient and family: identification of a health proxy or surrogate decision maker; identification of pre-existing advanced directives, living will, or DNR; understanding of family and social circumstances that may affect decision making; and an assessment of prognosis. Following this assessment, it's important to document patient wishes, being pain and symptom management and family support, continue full trauma care unless as consistent with patient's wishes, and begin a palliative screen based on injuries, frailty, possible outcomes, and administration of the "surprise question." The "surprise question" is: "Would you be surprised if the patient were dead in 12 months?" has been validated in a variety of clinical conditions to be a reliable marker of palliative care needs (34). A response of "No, I would not be surprised," if applicable, should trigger palliative care pathways, advanced care planning discussions, and a clarification of goals of care. A second family meeting should be held within 72 hours to further solidify goals of care and offer timelimited trials when appropriate (2). At this time, it is also important to begin surrogate and healthcare proxy education by instructing them that their role is to make the decisions that the patient would want.

\section{Special consideration: geriatric trauma}

Nearly $25 \%$ of patients hospitalized for trauma are 65 years or older (35). Furthermore, the number of geriatric trauma patients continues to age as the United States population ages and medical advances keep adults active later in life. Geriatric patients are not simply older adults, but rather a growing subset of the population with complex and unique needs after trauma (36). In a study of older Medicare beneficiaries who died less than 6 months after trauma, Lilley et al. found that fewer than $5 \%$ received inpatient palliative care (3). For these older trauma patients, inpatient palliative care was infrequent and was largely limited to those who survived less than two weeks postdischarge. In this study, palliative care was associated with higher rates of discharge to hospice and reduced healthcare utilization at the end of life, suggesting that palliative care is beneficial, but underutilized, in this age group (3). TQIP also recommends a frailty screening for all geriatric trauma patients, and a positive screening should trigger palliative care pathways $(2,37)$.

Literature has shown increased patient comfort and decreased in-hospital complications when geriatrics consults are placed for geriatric trauma patients $(3,38)$. Geriatrics utilizes an interdisciplinary approach to perform a comprehensive geriatric assessment, address the needs of older patients, and identify the unique features of disease presentation, interactions between medications and safe medication prescribing (38). This simple consult can improve the post injury experience of older trauma patients and facilitate recovery and healing. 


\section{Emergency general surgery considerations}

Disproportionately, older adults are admitted for emergency general surgery diseases (39). For older patients who develop EGS, many have a baseline high illness burden with a combination of functional dependence, dementia, functional limitations, use of helpers, multimorbidity, and high healthcare utilization. For these patients, EGS is serious and life-threatening, and the risk is not limited to the surgical procedure alone $(40,41)$. In the year after surgery, older adults have a 30\% 1-year mortality and high rates of healthcare utilization, suggesting that even patients who survive the initial hospitalization are extremely vulnerable. Among those who survive, one in 10 older Americans who were community-dwelling transfer to a nursing facility or long-term acute care facility by nine months postoperatively (42). Again, the "surprise question" is a simple and useful tool to screen patients for palliative care needs to identify patients with a high risk of death. The "surprise question" was prospectively validated in 119 older emergency general surgery patients, where a surgeon response of "No, I would not be surprised" response within the first 36 hours of patient evaluation was associated with an increased odds of patient death in the subsequent year, with an odds ratio of 4.8 (95\% CI: 2.1-11.1) (43).

Unfortunately, qualitative studies on this population have suggested that patients who undergo these procedures tend to view the immediate decision for surgery as a binary lifeor-death decision whereby surgery is necessary to prolong life $(44,45)$. In addition, recovery after life-threatening EGS also does not appear to translate into a desire for future advanced care planning (45). Many opportunities exist to better define and integrate the use of palliative care routinely into the care of EGS patients not only in the acute setting but also post-discharge.

\section{Implementation}

Under current United States acute care surgery paradigms, palliative care is often initiated late in the course of a critical illness or not at all (1). While studies have shown that the integration of formal palliative care in the trauma bay or surgical ICU has been associated with increased rates of formalized advanced directives, decreased ICU length of stay, increased use of hospice, and decreased use of nonbeneficial life-sustaining therapies, the initiation and timing of palliative care consultation in trauma patients remains variable across hospital systems $(1,11,46,47)$.
Use of specific criteria to prompt proactive referral for palliative care consultation seems to help reduce utilization of ICU resources without changing mortality, while increasing involvement of palliative care specialists for critically ill patients and families in need (48). One promising strategy for implementation of palliative care in the ICU is to actively screen all ICU patients using predetermined criteria to "trigger" a palliative care consultation $(1,48)$. "Triggers" examined by several studies include: (I) ICU admission following a hospital stay greater than or equal to 10 days; (II) age greater than 80 with two or more life-threatening comorbidities (as defined by Acute Physiology and Chronic Health Evaluation II definitions of severe chronic organ insufficiency); (III) diagnosis of active stage IV malignancy; (IV) status post cardiac arrest; or (V) diagnosis of intracerebral hemorrhage requiring mechanical ventilation $(1,11)$. For institutions where culture is a barrier to proactive utilization of palliative care, these "triggers" are concrete measures which can be tracked and measured to increase buy-in.

\section{Conclusions}

There are many barriers that lead to underutilization of palliative care services in the acute surgical setting, which can stem from factors that stem from patient and surrogates, physicians, and systems (23). Patient factors include limited understanding of chronic and acute illness and prognosis, surrogate preparedness, emotions, and the patient's beliefs. Surgeon resistance can be related to prognostic uncertainty, lack of training in communication methods, discomfort or inexperience with palliative care, and an ethical construct to "do everything" (23). System barriers can include limited availability of palliative care specialists, local practice patterns, time constraints, and limited quality of evidence regarding outcomes.

Fortunately, an increasing emphasis on the standard and routine use of palliative care principles for all seriously trauma and emergency surgery patients may help lower barriers over time. An increased focus on risk evaluation with frailty screening and emerging science regarding communication strategies may help surgeons feel more comfortable with simultaneous use of palliative care in parallel with ongoing medical treatments, rather than using palliative care as a last resort for patients in whom medical or surgical therapies are no longer likely to prolong life. Routine utilization of primary care principles and specialty palliative care is essential to align goal-concordant 
treatments with surgical patient outcomes. Future research can strengthen the existing data with examination of outcomes, reduction of bias, and optimization of implementation.

\section{Acknowledgments}

Funding: This publication was made possible by the Clinical and Translational Science Collaborative of Cleveland, KL2TR002547 from the National Center for Advancing Translational Sciences (NCATS) component of the National Institutes of Health and NIH roadmap for Medical Research. Its contents are solely the responsibility of the authors and do not necessarily represent the official views of the NIH.

\section{Footnote}

Provenance and Peer Review: This article was commissioned by the Guest Editors (Fabian M. Johnston and Ana Berlin) for the series "Palliative Care and Surgery" published in Annals of Palliative Medicine. The article has undergone external peer review.

Reporting Checklist: The authors have completed the Narrative Review reporting checklist (available at https:// apm.amegroups.com/article/view/10.21037/apm-20-2428/ rc).

Conflicts of Interest: All authors have completed the ICMJE uniform disclosure form (available at https://apm. amegroups.com/article/view/10.21037/apm-20-2428/coif). The series "Palliative Care and Surgery" was commissioned by the editorial office without any funding or sponsorship. VPH is supported by the Clinical and Translational Science Collaborative of Cleveland (KL2TR002547) from the National Center for Advancing Translational Sciences (NCATS) component of the National Institutes of Health and NIH roadmap for Medical Research. VPH spouse is a consultant for Zimmer Biomet, Medtronic, Atricure, and Astra Zeneca. The authors have no other conflicts of interest to declare.

Ethical Statement: The authors are accountable for all aspects of the work in ensuring that questions related to the accuracy or integrity of any part of the work are appropriately investigated and resolved.
Open Access Statement: This is an Open Access article distributed in accordance with the Creative Commons Attribution-NonCommercial-NoDerivs 4.0 International License (CC BY-NC-ND 4.0), which permits the noncommercial replication and distribution of the article with the strict proviso that no changes or edits are made and the original work is properly cited (including links to both the formal publication through the relevant DOI and the license). See: https://creativecommons.org/licenses/by-nc-nd/4.0/.

\section{References}

1. Hua MS, Li G, Blinderman CD, et al. Estimates of the need for palliative care consultation across united states intensive care units using a trigger-based model. Am J Respir Crit Care Med 2014;189:428-36.

2. ACS TQIP Palliative Care Best Practice Guidelines. American College of Surgeons Committee on Trauma. Available online: https://www.facs.org/-/media/files/ quality-programs/trauma/tqip/palliative_guidelines.ashx. Accessed 12/3/2020.

3. Lilley EJ, Lee KC, Scott JW, et al. The impact of inpatient palliative care on end-of-life care among older trauma patients who die after hospital discharge. J Trauma Acute Care Surg 2018;85:992-8.

4. O'Connell K, Maier R. Palliative care in the trauma ICU. Curr Opin Crit Care 2016;22:584-90.

5. Adolph MD, Frier KA, Stawicki SP, et al. Palliative critical care in the intensive care unit: A 2011 perspective. Int J Crit Illn Inj Sci 2011;1:147-53.

6. Mosenthal AC, Weissman DE, Curtis JR, et al. Integrating palliative care in the surgical and trauma intensive care unit: a report from the Improving Palliative Care in the Intensive Care Unit (IPAL-ICU) Project Advisory Board and the Center to Advance Palliative Care. Crit Care Med 2012;40:1199-206.

7. Truog RD, Campbell ML, Curtis JR, et al. Recommendations for end-of-life care in the intensive care unit: a consensus statement by the American College corrected of Critical Care Medicine. Crit Care Med 2008;36:953-63.

8. Bradley CT, Brasel KJ. Developing guidelines that identify patients who would benefit from palliative care services in the surgical intensive care unit. Crit Care Med 2009;37:946-50.

9. Laupland KB, Kirkpatrick AW, Kortbeek JB, et al. Longterm mortality outcome associated with prolonged 
admission to the ICU. Chest 2006;129:954-9.

10. Lilly CM, De Meo DL, Sonna LA, et al. An intensive communication intervention for the critically ill. Am J Med 2000;109:469-75.

11. Norton SA, Hogan LA, Holloway RG, et al. Proactive palliative care in the medical intensive care unit: effects on length of stay for selected high-risk patients. Crit Care Med 2007;35:1530-5.

12. Bakitas M, Lyons KD, Hegel MT, et al. Effects of a palliative care intervention on clinical outcomes in patients with advanced cancer: the Project ENABLE II randomized controlled trial. JAMA 2009;302:741-9.

13. Puntillo K, Nelson JE, Weissman D, et al. Palliative care in the ICU: relief of pain, dyspnea, and thirst--a report from the IPAL-ICU Advisory Board. Intensive Care Med 2014;40:235-48.

14. Sharp JT, Drutz WS, Moisan T, et al. Postural relief of dyspnea in severe chronic obstructive pulmonary disease. Am Rev Respir Dis 1980;122:201-11.

15. Collis E, Mather H. Nausea and vomiting in palliative care. BMJ 2015;351:h6249.

16. Grassi L, Caraceni A, Mitchell AJ, et al. Management of delirium in palliative care: a review. Curr Psychiatry Rep 2015;17:550.

17. LeGrand SB. Delirium in palliative medicine: a review. J Pain Symptom Manage 2012;44:583-94.

18. Satsangi AK, Brugnoli MP. Anxiety and psychosomatic symptoms in palliative care: from neuro-psychobiological response to stress, to symptoms' management with clinical hypnosis and meditative states. Ann Palliat Med 2018;7:75-111.

19. Hsu CH, Chi CC, Chen PS, et al. The effects of aromatherapy massage on improvement of anxiety among patients receiving palliative care: A systematic review of randomized controlled trials. Medicine (Baltimore) 2019;98:e14720.

20. Pochard F, Darmon M, Fassier T, et al. Symptoms of anxiety and depression in family members of intensive care unit patients before discharge or death. A prospective multicenter study. J Crit Care 2005;20:90-6.

21. Tran LN, Back AL, Creutzfeldt CJ. Palliative Care Consultations in the Neuro-ICU: A Qualitative Study. Neurocrit Care 2016;25:266-72.

22. Bhangu JK, Young BT, Posillico S, et al. Goals of Care Discussions for the Imminently Dying Trauma Patient. J Surg Res 2020;246:269-73.

23. Cooper Z, Koritsanszky LA, Cauley CE, et al. Recommendations for Best Communication Practices to
Facilitate Goal-concordant Care for Seriously Ill Older Patients With Emergency Surgical Conditions. Ann Surg 2016;263:1-6.

24. Curlin FA, Nwodim C, Vance JL, et al. To die, to sleep: US physicians' religious and other objections to physicianassisted suicide, terminal sedation, and withdrawal of life support. Am J Hosp Palliat Care 2008;25:112-20.

25. Plaisier BR, Blostein PA, Hurt KJ, et al. Withholding/ withdrawal of life support in trauma patients: is there an age bias? Am Surg 2002;68:159-62.

26. Trunkey DD, Cahn RM, Lenfesty B, et al. Management of the geriatric trauma patient at risk of death: therapy withdrawal decision making. Arch Surg 2000;135:34-8.

27. Manara AR, Pittman JA, Braddon FE. Reasons for withdrawing treatment in patients receiving intensive care. Anaesthesia 1998;53:523-8.

28. Kirchhoff KT, Kowalkowski JA. Current practices for withdrawal of life support in intensive care units. Am J Crit Care 2010;19:532-41; quiz 542.

29. Kruser JM, Taylor LJ, Campbell TC, et al. "Best Case/Worst Case": Training Surgeons to Use a Novel Communication Tool for High-Risk Acute Surgical Problems. J Pain Symptom Manage 2017;53:711-719.e5.

30. Baile WF, Buckman R, Lenzi R, et al. SPIKES-A six-step protocol for delivering bad news: application to the patient with cancer. Oncologist 2000;5:302-11.

31. Hornor MA, Byrne JP, Engelhardt KE, et al. Examining racial disparities in the time to withdrawal of lifesustaining treatment in trauma. J Trauma Acute Care Surg 2018;84:590-7.

32. Haines KL, Jung HS, Zens T, et al. Barriers to Hospice Care in Trauma Patients: The Disparities in End-of-Life Care. Am J Hosp Palliat Care 2018;35:1081-4.

33. Weissman DE, Meier DE. Identifying patients in need of a palliative care assessment in the hospital setting: a consensus report from the Center to Advance Palliative Care. J Palliat Med 2011;14:17-23.

34. Jennings KS, Marks S, Lum HD. The Surprise Question as a Prognostic Tool \#360. J Palliat Med 2018;21:1529-30.

35. Bonne S, Schuerer DJ. Trauma in the older adult: epidemiology and evolving geriatric trauma principles. Clin Geriatr Med 2013;29:137-50.

36. Bradburn EH, Gross BW, Jammula S, et al. Improved outcomes in elderly trauma patients with the implementation of two innovative geriatric-specific protocols-Final report. J Trauma Acute Care Surg 2018;84:301-7.

37. Morley JE, Malmstrom TK, Miller DK. A simple frailty 
questionnaire (FRAIL) predicts outcomes in middle aged African Americans. J Nutr Health Aging 2012;16:601-8.

38. Ritchie CS, Kelley AS, Stijacic Cenzer I, et al. High Levels of Geriatric Palliative Care Needs in Hip Fracture Patients Before the Hip Fracture. J Pain Symptom Manage 2016;52:533-8.

39. Gale SC, Shafi S, Dombrovskiy VY, et al. The public health burden of emergency general surgery in the United States: A 10-year analysis of the Nationwide Inpatient Sample--2001 to 2010. J Trauma Acute Care Surg 2014;77:202-8.

40. Cooper Z, Lilley EJ, Bollens-Lund E, et al. High Burden of Palliative Care Needs of Older Adults During Emergency Major Abdominal Surgery. J Am Geriatr Soc 2018;66:2072-8.

41. Sokas C, Lee KC, Sturgeon D, et al. Preoperative Frailty Status and Intensity of End-of-Life Care Among Older Adults After Emergency Surgery. J Pain Symptom Manage 2021;62:66-74.e3.

42. Smith JW, Knight Davis J, Quatman-Yates CC, et al. Loss of Community-Dwelling Status Among Survivors of HighAcuity Emergency General Surgery Disease. J Am Geriatr Soc 2019;67:2289-97.

Cite this article as: Ferre AC, DeMario BS, Ho VP. Narrative review of palliative care in trauma and emergency general surgery. Ann Palliat Med 2022;11(2):936-946. doi: 10.21037/apm20-2428
43. Lilley EJ, Gemunden SA, Kristo G, et al. Utility of the "Surprise" Question in Predicting Survival among Older Patients with Acute Surgical Conditions. J Palliat Med 2017;20:420-3.

44. Nabozny MJ, Kruser JM, Steffens NM, et al. Constructing High-stakes Surgical Decisions: It's Better to Die Trying. Ann Surg 2016;263:64-70.

45. Sokas C, Yeh IM, Coogan K, et al. Older Adult Perspectives on Medical Decision Making and Emergency General Surgery: "It had to be Done." J Pain Symptom Manage 2021;61:948-54.

46. Campbell ML, Guzman JA. Impact of a proactive approach to improve end-of-life care in a medical ICU. Chest 2003;123:266-71.

47. O'Mahony S, McHenry J, Blank AE, et al. Preliminary report of the integration of a palliative care team into an intensive care unit. Palliat Med 2010;24:154-65.

48. Nelson JE, Curtis JR, Mulkerin C, et al. Choosing and using screening criteria for palliative care consultation in the ICU: a report from the Improving Palliative Care in the ICU (IPAL-ICU) Advisory Board. Crit Care Med 2013;41:2318-27. 